\title{
MatemaTIC: más allá de enseñar matemáticas en educación superior virtual
}

Alexander Rincón-Rojas ${ }^{1}$

Universidad Autónoma de Manizales

a.rincon@autonoma.edu.co

DOI: https://doi.org/10.21158/2357514x.v8.n2.2020.2746

Cómo citar este artículo: Rincón-Rojas, A. (2020). MatemaTIC: más allá de enseñar matemáticas en educación superior virtual. Revista Virtu@Imente, 8(2), 47-66.

DOI: https://doi.org/10.21158/2357514x.v8.n2.2020.2746

Fecha de recepción: 08 de agosto de 2020

Fecha de aprobación: 06 de octubre de 2020

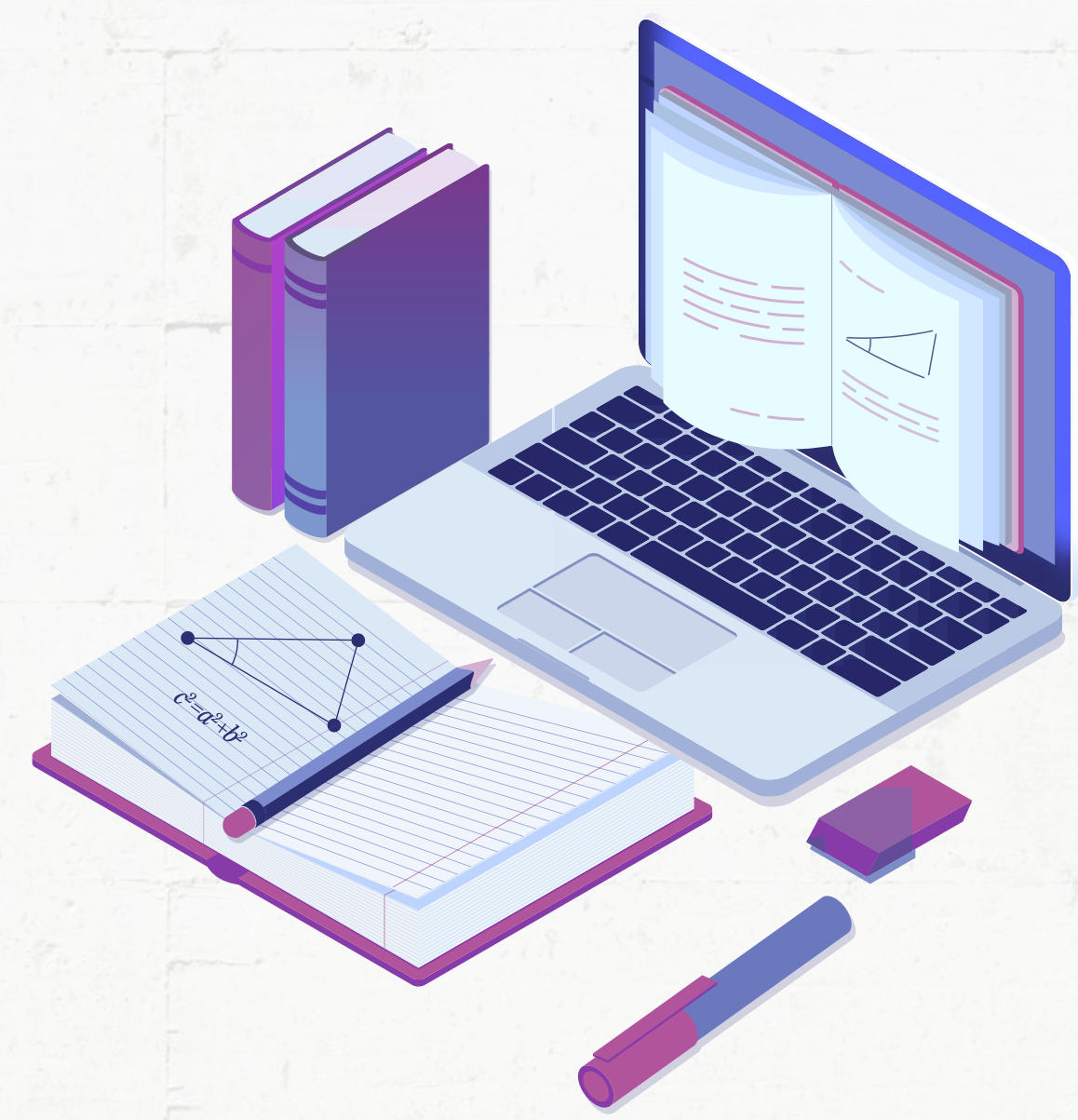

${ }^{1}$ Licenciado en Matemáticas - Universidad Distrital Francisco José de Caldas. Magister en Ciencias Matemáticas - Universidad Nacional de Colombia. Magister en Pedagogía - Universidad de la Sabana. ORCID: https://orcid.org/0000-0002-8156-4806.

(c) $\mathbb{D} \Theta \Theta$ 


\section{RESUMEN}

En el presente artículo se describe MatemaTIC como una estrategia académica para la consolidación y el fortalecimiento de las competencias matemáticas en el ámbito profesional, enmarcada dentro de lineamientos institucionales; que además se convierte en un escenario de vinculación y retención para los estudiantes en educación superior a distancia mediada por TIC. Los resultados emergen de un proceso investigativo de carácter cualitativo y de corte descriptivo, realizado durante varios periodos académicos con estudiantes que se ubican en diferentes regiones del país, en condiciones socioculturales diferentes; estos se enfocan en mostrar la estrategia dentro de una estructura didáctica institucional y algunas implicaciones de su realización. MatemaTIC se estructuro a partir de los lineamientos del modelo de entornos convergentes y se desarrolló en cuatro fases. Al final, se evidencia como MatemaTIC, mediante las situaciones utilizadas en las secuencias didácticas digitales están en sintonía con los problemas profesionales o académicos de su área. Por lo que esta relación hace que los estudiantes, por un lado, establezcan nexos funcionales entre el campo teórico de las matemáticas y los campos de aplicación -Administración de Empresas-, y, por el otro, que durante los diseños de las unidades didácticas contextuales el profesor deba actualizar de forma permanente sus conocimientos más allá de lo disciplinar.

Palabras clave: educación superior a distancia; competencias matemáticas; enseñanza de matemáticas; modelo de entornos convergentes; secuencias didácticas digitales; unidades didácticas contextuales; estrategia académica; inclusión educativa. 


\section{MatemaTIC: \\ beyond teaching mathematics in virtual higher education}

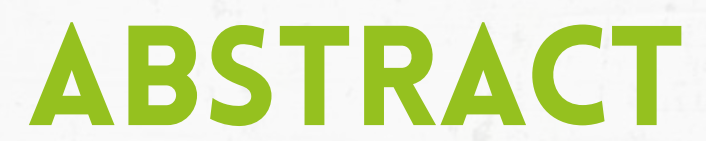

This article describes MatemaTIC as an academic strategy for the consolidation and strengthening of mathematical competencies in the professional field, framed within institutional guidelines; which also becomes a linking and retaining scenario for students in distance higher education mediated by ICTs. The results emerge from a qualitative and descriptive research process, carried out during several academic periods with students who are located in different regions of the country, and in different socio-cultural conditions; and they are focused on showing the strategy within an institutional didactic structure and some implications of its implementation. MatemaTIC was structured based on the guidelines of the converged environments model and was developed in four phases. At the end, it is clear how MatemaTIC, through the situations used in the digital teaching sequences, is in tune with the professional or academic problems in the area. On the one hand, this relationship makes students establish functional links between the theoretical field of mathematics and the fields of application -Business Administrationand makes it necessary for the teacher to permanently update his knowledge beyond the discipline during the designs of the contextual didactic units.

Keywords: higher distance education; mathematical competences; mathematics instruction; Converged Environments Model; digital didactic sequences; contextual didactic units; academic strategy; educational inclusion. 


\section{MatemaTIC: muito além de ensinar matemática no Ensino Superior virtual}

\section{RESUMO}

Neste artigo, o MatemaTIC é descrito como uma estratégia acadêmica para a consolidação e o fortalecimento das competências matemáticas no âmbito profissional, enquadrada em diretrizes institucionais; que também se torna um ambiente de vinculação e retenção de alunos do Ensino Superior a distância mediado pelas TIC. Os resultados emergem de um processo de pesquisa qualitativa e descritiva, realizada ao longo de vários períodos letivos com alunos que se encontram em diferentes regiões do país e em condições socioculturais diferentes. Esses resultados, concentram-se em mostrar a estratégia dentro de uma estrutura didática institucional e algumas implicações de sua realização. O MatemaTIC foi estruturado com base nas diretrizes do modelo de ambientes convergentes e foi desenvolvido em quatro fases. Ao final, constata-se que o MatemaTIC, por meio das situações utilizadas nas sequências didáticas digitais, está em sintonia com os problemas profissionais ou acadêmicos de sua área. Portanto, esta relação faz com que os alunos, por um lado, estabeleçam vínculos funcionais entre o campo teórico da matemática e os campos de aplicação —Administração de Empresas—, e, por outro, que durante os desenhos das unidades didáticas contextuais o professor deve atualizar permanentemente seus conhecimentos muito além da disciplina.

Palavras-chave: educação superior a distância; habilidades matemáticas; ensino de matemática; modelo de ambientes convergentes; sequências didáticas digitais; unidades didáticas contextuais; estratégia acadêmica; inclusão educacional. 


\section{MatemaTIC: \\ l'enseignement virtuel des mathématiques en milieu universitaire}

\section{RÉSUMẼ}

Cetarticle présente MatemaTIC, une stratégie académique pour la consolidation et le renforcement des compétences mathématique en milieu professionnel, encadrée par des directrices institutionnelles, qui se transforme, grâce aux $\mathrm{TIC}$, en modèle de captation et de rétention des étudiants de modalité non présentielle. Les résultats de cette étude sont issus d'un processus de recherche qualitatif et descriptif, mené sur plusieurs périodes académiques, avec le concours d'étudiants de catégories sociales et régions diffèrentes, et qui présenteront la stratégie et les applications mises en oeuvre au sein d'une structure didactique institutionnelle. MatemaTIC est structuré sur la base de lignes directrices de modèles d'environnements convergents et développé en quatre phases. Ce modèle se révèle être en phase avec les problématiques professionnelles et académiques des apprenants à la lumière des séquences pédagogiques de type numérique mise en place. Cette situation amène les étudiants à établir, d'une part, des liens fonctionnels entre le domaine théorique des mathématiques et les domaines d'application réels gestion d'entreprise -, et, d'autre part, oblige les enseignants à actualiser constamment leurs connaissances pour concevoir des unités didactiques contextualisées

Mots-clés: enseignement supérieur à distance; compétences mathématiques; enseignement des mathématiques; modèle d'environnement convergent; séquences d'enseignement numériques; unités d'enseignement contextualisées; stratégie académique; inclusion éducative. 


\section{Introducción}

MatemaTIC nace como una alternativa para la enseñanza, el aprendizaje y la evaluación de las matemáticas en el contexto de la educación superior a distancia mediada por Tecnologías de la Información y la Comunicación (TIC). Bajo el modelo de entornos convergentes (MEC-Complexus), desarrollado por el Departamento de Estudios a Distancia en el marco de la misión institucional de la Universidad Autónoma de Manizales, esta busca, por un lado, promover el fortalecimiento de las competencias matemáticas en el ámbito profesional y, por el otro, afianzarse como un escenario para la vinculación y permanencia de los estudiantes en la educación universitaria.

MatemaTIC es el resultado de un proceso investigativo, de corte cualitativo, con alcance descriptivo, que se hace a partir de diferentes análisis contextuales y categóricos que se han llevado a cabo durante diferentes periodos académicos, estableciendo sus alcances y limitaciones en cuanto a su desarrollo conceptual y procedimental. Por pertinencia, solo se presentan los resultados de un ciclo académico.

Este artículo debe considerarse resultado parcial de un producto de mayor envergadura, que contempla, entre otros análisis: las transiciones experimentadas en las situaciones problémicas abordadas en las diferentes versiones de MatemaTIC, en el marco del modelo de entornos convergentes; la caracterización de la población atendida cantidad, regiones, género, edad, continuidad, promoción, deserción y resultados externos, entre otros-, y la medición del impacto en el acompañamiento y seguimiento al logro a través de actividades cognitivas y metacognitivas.
Así, el presente artículo se estructura en cinco partes fundamentales: en la primera, se establecen los contextos problémicos y de justificación del estudio parcial, hasta proponer una pregunta de investigación que gira en torno a la búsqueda de alternativas para el desarrollo de competencias matemáticas en educación superior a distancia mediada por TIC, y a la generación de escenarios de vinculación con la vida académica. La segunda parte está dedicada a los postulados conceptuales que guían el estudio. La tercera, a la presentación metodológica en el marco de unos lineamientos institucionales y didácticos. Estos llevan a la cuarta parte, que se dedica a sintetizar algunos resultados. El documento finaliza con algunas conclusiones. Se reitera que MatemaTIC es una propuesta no finalizada de un proceso reflexivo adelantado en el interior del Departamento de Estudios a Distancia de la Universidad Autónoma de Manizales.

\section{Contexto problémico y justificación}

La consolidación de MatemaTIC emerge de al menos seis aspectos contextuales que, aunque son transferibles a otras modalidades de estudio, son relevantes para el modelo de educación a distancia mediada por TIC. Estos se mencionan a continuación. 
El primer aspecto proviene de la necesidad de articular los diferentes niveles educativos de acuerdo con las orientaciones de política pública emitidas por el Ministerio de Educación Nacional de Colombia, que expresa, entre otras cosas:

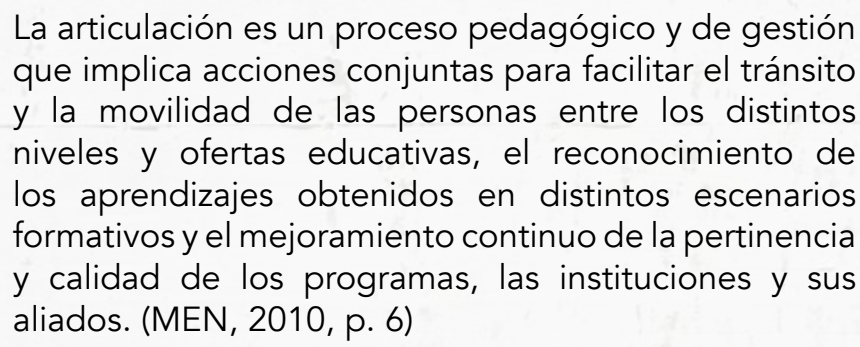
que implica acciones conjuntas para facilitar el tránsito y la movilidad de las personas entre los distintos niveles y ofertas educativas, el reconocimiento de los aprendizajes obtenidos en distintos escenarios formativos y el mejoramiento continuo de la pertinencia y calidad de los programas, las instituciones y sus aliados. (MEN, 2010, p. 6)

En este sentido, las diferentes instituciones educativas, y en particular la universidad, deben establecer estrategias y mecanismos para promover los aprendizajes y la continuidad dentro del sistema educativo, garantizando el avance en los procesos formativos y buscando:

\begin{abstract}
Un diálogo que implica construir sinergia y unidad en lo pedagógico alrededor de las competencias profesionales, como eje de la formación, y asumir una transformación en los distintos ámbitos de la gestión institucional para hacerla posible, darle sostenibilidad y evaluarla continuamente. (MEN, 2010, p. 7)
\end{abstract}

Lo anterior implica que se deben construir puentes entre la educación media y la universitaria que, en línea con los propósitos de formación profesional, acojan a los estudiantes y los nivelen en el éxito; no en el fracaso, como suele ocurrir en el medio universitario, en donde al ingresar a los programas y no tener las herramientas académicas necesarias, se excluye a los estudiantes de una u otra forma.
Un segundo aspecto, relacionado con el anterior, radica en la necesidad de establecer componentes y estrategias que permitan vincular a los estudiantes a la educación superior a distancia mediante el uso adecuado de herramientas informáticas para aprender a aprender. Esta necesidad contempla no solo el acceso a los medios físicos, sino a una alfabetización digital, que conlleva el desarrollo de competencias en el tratamiento de la información y la producción y evaluación de contenidos, en condiciones de criticidad y objetividad. De igual forma, el acceso a las redes informáticas deberá ofrecer perspectivas de trabajo diferentes a las tradicionales, dando apertura al mundo y permitiendo «la comunicación entre las personas eliminando las barreras de espacio y del tiempo, de identidad y de estatus» (Segura, 2011, p. 95).

De igual manera, es indispensable incorporar el uso de las TIC en las cadenas de producción bajo las condiciones establecidas por Jaime Yanes, quien afirma que: «La incorporación de las TIC en la actual producción ha permitido que los activos más valiosos de una empresa ya no sean los tangibles, sino los activos intangibles como los conocimientos individuales y organizacionales» (2014, p. 49). Así, se pone de manifiesto que el uso de las TIC no debe ser el fin, sino el medio para la consecución de las metas establecidas en el entorno educativo.

Un tercer aspecto se refiere al tipo de población a la que se dirige MatemaTIC. La universidad, a través del Departamento de Estudios a Distancia, oferta dos programas de formación tecnológica -Tecnología en Gestión de Negocios y 
Tecnología en Gestión de Empresas Agroindustriales_y uno profesional —Administración de Empresas-, que se imparten a estudiantes ubicados en diferentes regiones del país y con condiciones sociodemográficas diversas.

A continuación, en la figura 1 se muestran los estudiantes que estuvieron en MatemaTIC durante el periodo académico 20181 y renovaron matrícula para el 2018-2.

Figura 1. Distribución demográfica de estudiantes por regiones y sexo para el periodo 2018-2 después de MatemaTIC

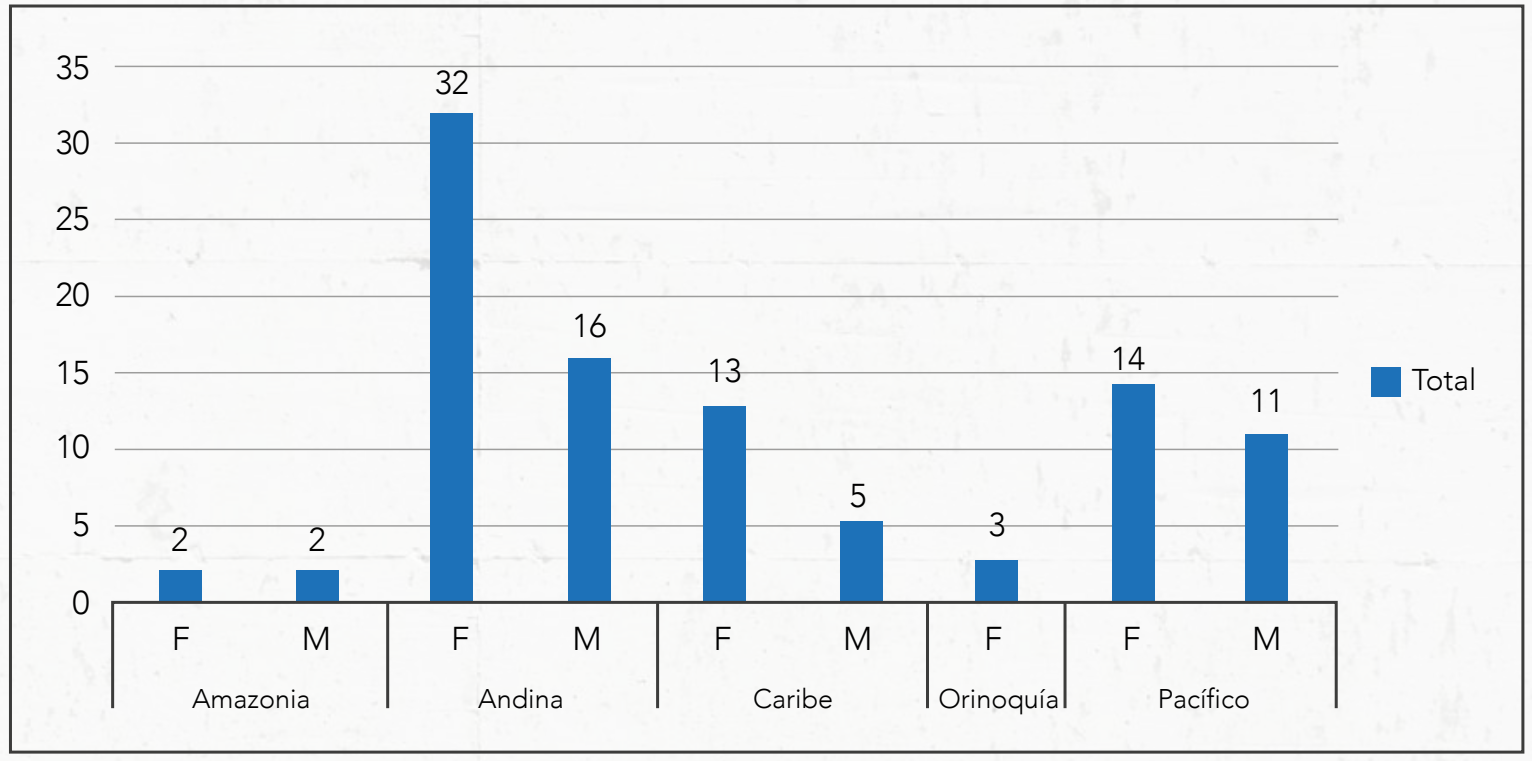

Fuente. Elaboración propia.

El grafico nos lleva a reflexionar y a reconocer que los estudiantes tienen diferencias sociales y culturales, que de una $u$ otra forma influyen en la permanencia y calidad de los procesos formativos. Por lo tanto, es indispensable que los programas que brinda la universidad generen estrategias de inclusión y promuevan la participación en la construcción del conocimiento en condiciones de favorabilidad para aquellos estudiantes vulnerables, sin que ello implique una desmejora en la calidad de los procesos. Frente a lo anterior, Sarrionandia y Duk expresan: 
Avanzar hacia la inclusión supone, por tanto, reducir las barreras de distinta índole que impiden o dificultan el acceso, la participación y el aprendizaje, con especial atención en los alumnos más vulnerables o desfavorecidos, por ser los que están más expuestos a situaciones de exclusión y los que más necesitan de la educación, de una buena educación. $(2008$, p. 1)

Un cuarto aspecto hace referencia al nivel de competencia en matemáticas con el que ingresan los estudiantes a la formación tecnológica y profesional en el modelo de educación a distancia. Al ser los programas ofertados por la universidad a través del Departamento de Estudios a Distancia una estrategia institucional para el fortalecimiento y desarrollo regional sostenible que busca integrar a diferentes personas, en donde los marcadores de desempeño de ingreso son aparentemente bajos con relación a la media nacional, en la figura 2 se muestra la relación de ingreso dado por las pruebas Saber 11 de los estudiantes de la cohorte 2018-1 que cursaron MatemaTIC.

Figura 2. Resultado en matemáticas en las pruebas Saber 11 de ingreso a la educación superior

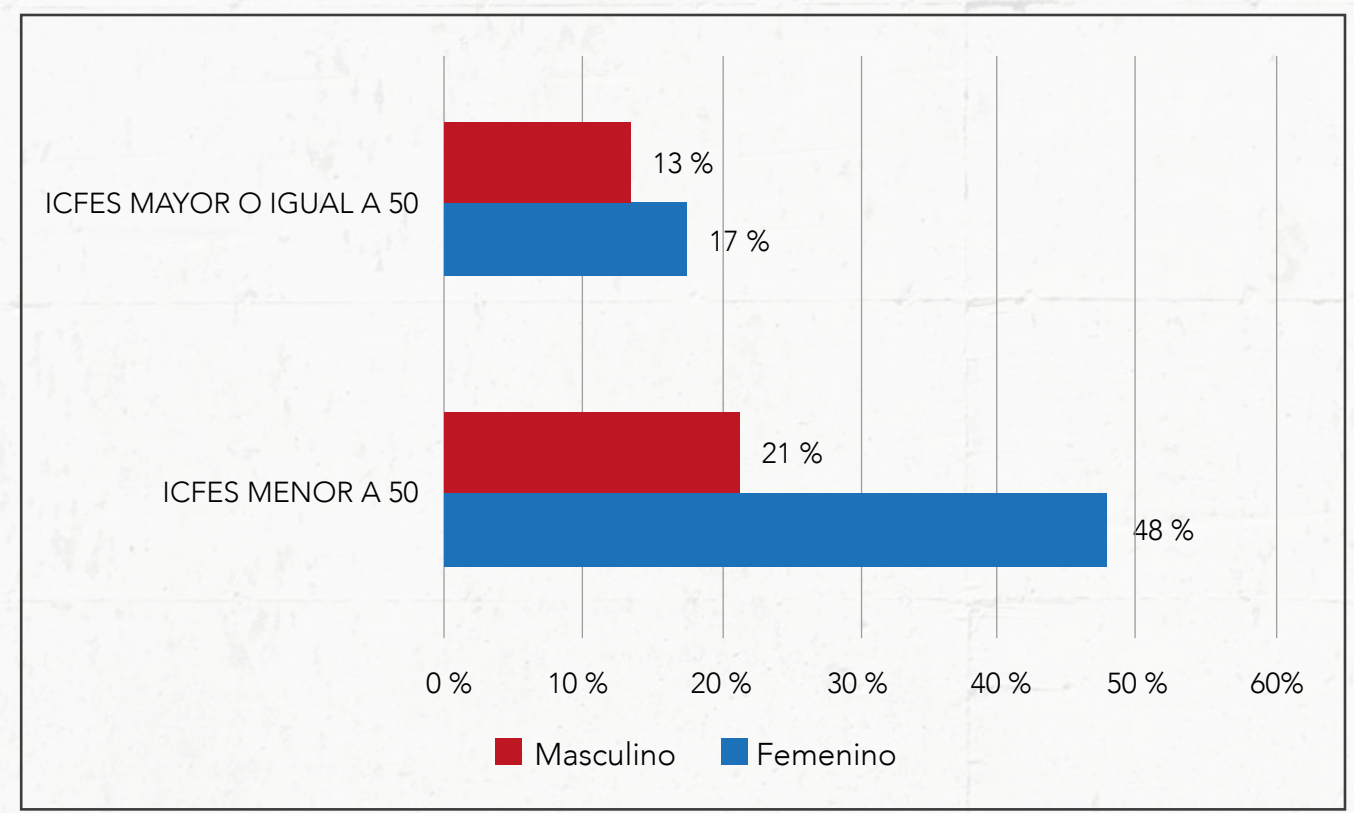

Fuente. Elaboración propia. 
Esto pone en evidencia que para avanzar en el proceso de apropiación de competencias en matemáticas es fundamental construir estrategias contextuales de enseñanza, aprendizaje y evaluación acordes a las necesidades académicas, e identificar los posibles obstáculos en el aprendizaje que poseen los estudiantes al ingresar en el modelo de educación a distancia.

De igual forma, es imprescindible que las estrategias diseñadas e implementadas respondan a una transformación progresiva del pensamiento matemático o, en palabras de Dávila, Borrachero, Cañada y Sánchez, que expresen: «La necesidad de modificar, transformar gradualmente estos conceptos existentes en conceptos científicos más cercanos para que se produzca un aprendizaje significativo a través del cambio conceptual» (2018, p. 93). Una vez hecho este proceso, es necesario introducir estratégicamente aspectos generales de las matemáticas que nivelen, solventen los problemas identificados e introduzcan a escenarios de mayor complejidad.

Un quinto aspecto por relacionar establece la necesidad de implementar procesos metacognitivos en la enseñanza y el aprendizaje que se realizan en la universidad, que posibiliten e incentiven el aprendizaje autónomo, autodirigido y regulado, fijando como meta continua "el aprender a aprender» para el desarrollo personal y grupal. En este sentido, como lo expresan Melgar y Elisondo: "Los estudiantes desarrollan procesos metacognitivos cuando pueden identificar objetivos de aprendizaje y realizar las correspondientes acciones y estrategias para lograrlos. Los procesos metacognitivos implican reflexiones y autorregulaciones permanentes de la comprensión (2017, p. 19)», posibilitando así un mejoramiento continuo de su ser y de su futura profesión, lo que los sitúa como protagonistas de su evolución y futura evaluación de su desempeño.

Por último, al establecer algunas de las necesidades académicas en el ámbito del aprendizaje de las matemáticas, se ha posibilitado realizar lecturas de contextos mediante el diálogo permanente entre los diferentes actores inmersos en el proceso de enseñanza, aprendizaje y evaluación del conocimiento escolar matemático en función de las competencias profesionales por desarrollar. En este sentido, el conocimiento escolar matemático se transforma en ayudante de los procesos de formación profesional y el proceso de «aprender, desde el punto de vista sociocultural, se relaciona con cómo las personas se apropian de herramientas para pensar y actuar en una comunidad de práctica» (Linares, 2008, p. 7), haciendo énfasis en su futura vida profesional y laboral.

De lo anterior se puede destacar que MatemaTIC es una estrategia no finalizada que se transforma internamente de acuerdo con las dinámicas externas y contextuales de la población en el marco de una política institucional regida por enfoques nacionales y regionales, lo que la sitúa como una propuesta de innovación abierta y de cocreación.

Teniendo en cuenta los aspectos mencionados, surge la pregunta: ¿cómo diseñar e implementar una estrategia académica que posibilite el fortalecimiento de competencias en matemáticas en el ámbito profesional y que sea un escenario de vinculación a la educación universitaria? 


\section{Marco conceptual}

En el marco conceptual, siguiendo las orientaciones del modelo de entornos convergentes (MEC-Complexus), se establecen las consideraciones didácticas provenientes de la educación matemática y de los contextos de los estudiantes.

Las orientaciones que aporta el MEC-Complexus a MatemaTIC devienen de una construcción, producto de las discusiones y aportes de los miembros del grupo de investigación. A continuación, se establecen algunos de los postulados más relevantes. Para profundizar al respecto, se recomienda al lector remitirse a los documentos del grupo de investigación en mención.

La educación superior a distancia se asume como una actividad de carácter procesual y social, donde las TIC median las interrelaciones e interacciones entre los diferentes actores, saberes y escenarios de aprendizaje intencionados. Estas orientaciones son acordes a los lineamientos institucionales que motivan la enseñanza y el aprendizaje sobre una base socioconstructivista, de base problémica, que reconocen los postulados del cambio representacional para que los estudiantes comprendan los fenómenos a través de las múltiples relaciones dialógicas que se desarrollan en los ambientes de aprendizaje virtuales y no virtuales, los cuales procuran ser socialmente relevantes.
El sistema de estudios a distancia de la universidad se rige bajo los principios de: «autodelimitación», que articula tres niveles contextuales en educación superior macrocontexto, políticas públicas en educación a distancia; mesocontexto, relacionado con la convergencia tecnológica, y microcontexto, referido a la práctica escolar-; «articulación interna», que lo sitúa como un sistema adaptativo complejo, donde la cohesión interna de las partes se regula e impulsa; «estratificación», que refiere a los procesos relacionales interacción e interactividad- entre las partes del sistema; y, por último, el principio de "evolución», que ubica al sistema de forma dinámica frente a las incertidumbres internas y externas, convirtiéndolo en una estrategia de proyección social que aporta al desarrollo regional sostenible (Ágamez, Daza y Rincón, 2018, p. 66).

Una vez catalogado el sistema de estudios a distancia, de forma paralela se constituye el modelo de entornos convergentes (MEC-Complexus), que es el encargado de guiar el proceso de formación mediante la integración, por un lado, de los entornos de aprendizaje y, por el otro, de las unidades didácticas contextualizadas para luego, materializarse en secuencias didácticas digitales. 
Figura 3. Secuencia didáctica digital como emergencia de las Unidades Didácticas Contextualizadas y los entornos de aprendizaje

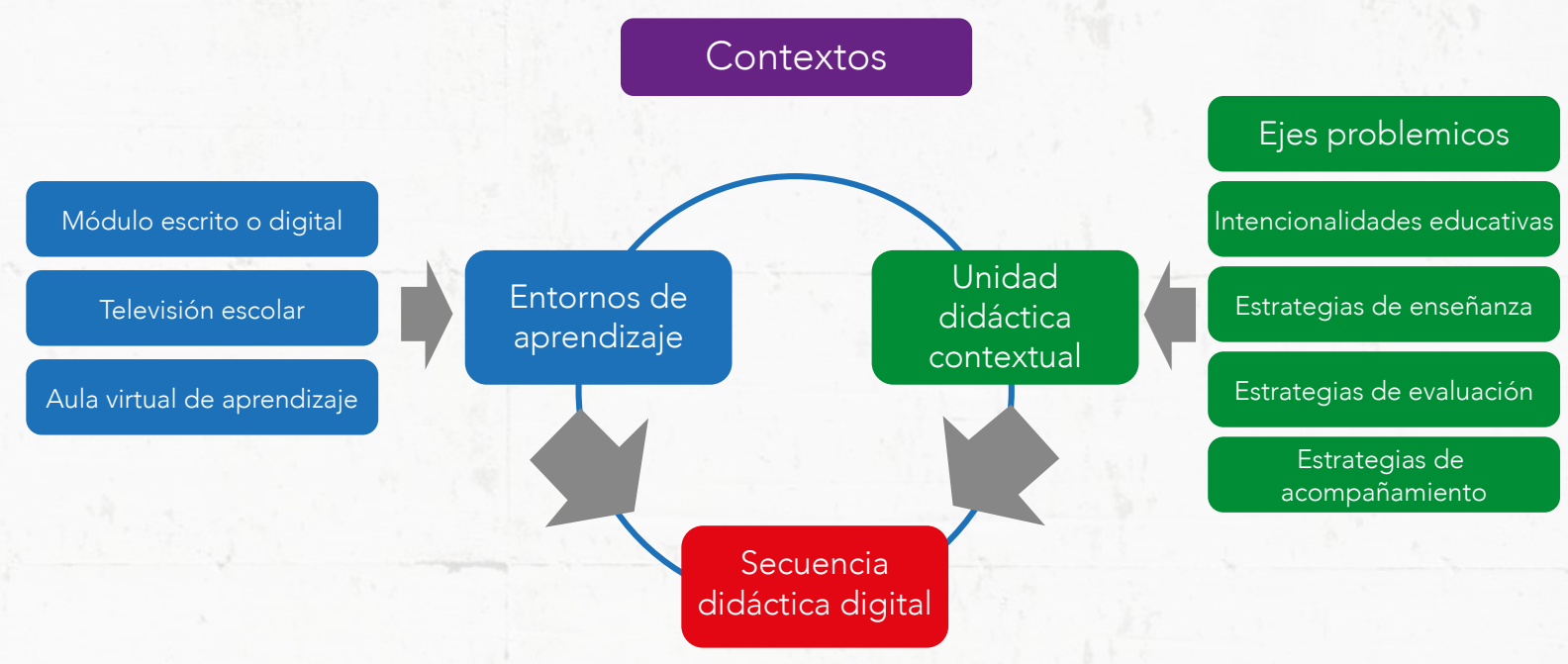

Fuente. Elaboración propia.

Los entornos de aprendizaje son los espacios formativos mediante los cuales se desarrollan los procesos comunicativos y de interacción social. Estos son de tres tipos: el material didáctico -impreso y digital_, donde se recopilan los principales contenidos, conceptos y procedimientos que debe conocer el estudiante para un adecuado desarrollo teórico presente y futuro; la televisión escolar, que refiere a espacios para encuentros sincrónicos entre el profesor titular, el profesor asistente y los estudiantes que están en las regiones, a través de emisiones de televisión satelital en vivo; $y$, por último, las aulas virtuales de aprendizaje, que se caracterizan por superar la idea de los repositorios de información para convertirse en sitios de interacción y de aprendizaje guiado, desarrollado de forma autónoma y colaborativa.
Las Unidades Didácticas Contextualizadas (UDC) son estructuras dinámicas que orientan y dosifican el aprendizaje, la enseñanza y la evaluación de las intencionalidades educativas, a través de un conjunto de actividades mediadas por las interacciones con el docente, las cuales se enmarcan en un contexto problémico profesional.

Dentro de las UDC se identifican los siguientes componentes: la definición de ejes problémicos de la profesión, que guían el trabajo docente y de problematización de los contenidos; el establecimiento de las intencionalidades educativas, que se expresan en términos de competencias genéricas y profesionales; la estrategia de enseñanza, que integra elementos cognitivos y metacognitivos para el 
adecuado desarrollo del proceso formativo; la estructuración de la estrategia de evaluación, que contempla elementos de promoción y de formación; y, para finalizar, la estrategia de acompañamiento, que establece las condiciones de seguimiento, asesoramiento y trabajo individual para garantizar el logro. Estos componentes se organizan en los ejes de acción.

Los ejes de acción se instauran bajo las consideraciones de la teoría de situaciones didácticas de Brousseau y son los encargados de coordinar los componentes de la UDC. Estos ejes se estructuran en: un momento de indagación, que explora las teorías implícitas con que los estudiantes ingresan frente a unos problemas modelados o estandarizados; un momento de problematización, en el cual se les presentan situaciones relacionados con los ejes problémicos de la profesión; un momento de tematización, que aporta los contenidos problematizados y de la asignatura; un momento de aplicación, cuando se responden los problemas planteados y se establecen los criterios de solución sobre una base teórica, y el momento de finalización, que transfiere el conocimiento a otros escenarios problémicos de igual o mayor complejidad.

Como consecuencia, aparecen las secuencias didácticas digitales (SDD), que materializan y dinamizan las UDC y los entornos de aprendizaje entorno a la resolución de situaciones problémicas de la profesión.

Por otro lado, MatemaTIC incorpora los lineamientos de los estándares básicos de competencias en matemáticas del Ministerio de Educación Nacional de Colombia (MEN) que establece: 
Figura 4. Relación sistémica del pensamiento matemático

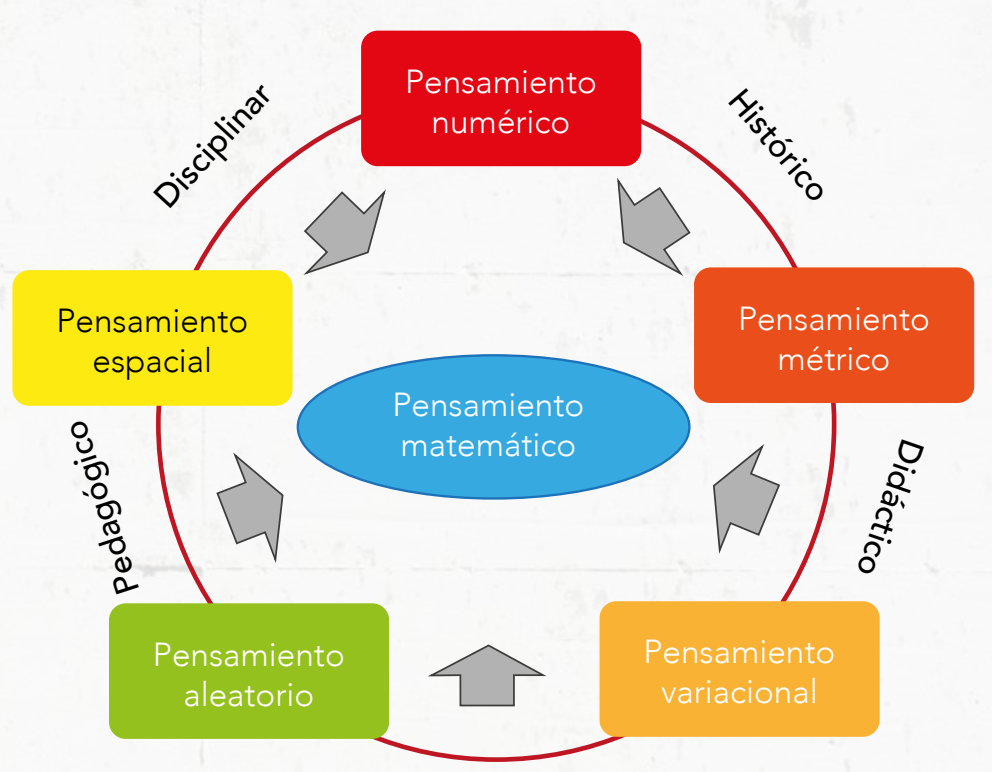

\section{Resolución de problemas}

Fuente. Elaboración propia.

El pensamiento matemático es sistémico y articulado por el desarrollo de otros subpensamientos —numérico, métrico, variacional, aleatorio y espacial-, los cuales, a través de un tratamiento desde lo pedagógico, didáctico, histórico y disciplinar, posibilitan la enseñanza y el aprendizaje de las matemáticas.

Históricamente se ha dado particular importancia al aprendizaje de las matemáticas, según el MEN (2006), debido a que varias concepciones que se tienen de ella; por ejemplo, su aplicabilidad en diferentes aspectos sociales y culturales, su asociación con el desarrollo del pensamiento lógico de las personas y porque se ha considerado a las matemáticas un elemento esencial para el desarrollo de la ciencia y la tecnología.

Durante gran parte del siglo XX las matemáticas fueron vistas como un cuerpo estable e infalible de verdades absolutas, lo que condujo a suponer que solo se requería estudiar, ejercitar y recordar un listado más o menos largo 
de contenidos, hechos, definiciones, propiedades de objetos matemáticos, axiomas, teoremas y procedimientos algorítmicos para formar a todos los estudiantes en el razonamiento lógico y en los conocimientos matemáticos.

En los últimos años, estas ideas han venido evolucionando. Se ha planteado que el desarrollo del pensamiento lógico y de competencias en los ámbitos tecnológico y científico no es atribuible a una determinada ciencia —en este caso, a las matemáticas-, sino que, por el contrario, es el resultado de una educación integral, de calidad, cuyo elemento esencial no sea un aprendizaje memorístico, sino el aprendizaje significativo. Esto en línea con lo que desarrolla Coll (1988), quien, grosso modo, afirma que un estudiante aprende un contenido cualquiera, un concepto, una explicación, un fenómeno, un procedimiento, una norma, etc., cuando es capaz de atribuirle un significado (p. 134). Por tal motivo es fundamental que el proceso enseñanza-aprendizaje busque ser lo más significativo posible para los estudiantes.

El MEN, en Estándares básicos de competencias en matemáticas (2006), plantea cinco procesos generales presentes en toda la actividad de este tipo que hacen que cualquier persona se considere matemáticamente competente. Estos son: formular y resolver problemas; modelar procesos y fenómenos de la realidad; comunicar; razonar, formular, comparar y ejercitar procedimientos y algoritmos. Cada uno de estos procesos prepara al estudiante para afrontar desafíos cuantitativos, pero no de forma aislada de la realidad y de otras ciencias, más bien, son coadyuvantes en la construcción e interpretación de las múltiples realidades desde enfoques y perspectivas diferentes.

\section{Metodología}

El presente artículo es resultado de un proceso de reflexión que se ha adelantado en diferentes periodos académicos, desde el año 2010, con estudiantes adscritos a los programas ofertados por la universidad a través del Departamento de Estudios a Distancia. Para los fines pertinentes, se presentan los resultados obtenidos durante la ejecución del programa MatemaTIC en el periodo académico 2018-1, con 98 estudiantes, en el cual se incorporó el modelo de entornos convergentes a la dinámica del programa.

Para este momento es preciso aclarar que MatemaTIC no hace parte de los planes de estudio de los programas de formación impartidos, por tanto, es una asignatura de libre opción dentro de la oferta de la universidad.

MatemaTIC se estructuró a partir de los lineamientos del modelo de entornos convergentes y se desarrolló en cuatro fases, que se presentan a continuación:

La primera fase evaluó los propósitos de formación frente a los contextos institucionales, incluyendo el profesional; los regionales, en cuanto a las características sociodemográficas de los estudiantes, y los niveles de competencia en matemáticas con que ingresan comparados con los de salida. Durante esta fase se establecen las pruebas estandarizadas de entrada para los estudiantes, de tal forma que se puedan fijar los puntos de partida. De igual manera, se determinan los principales ejes problémicos de la profesión, para construir o establecer los contextos de las situaciones problémicas y adecuar los contenidos. 
La segunda fase incorpora las situaciones problémicas en los entornos de aprendizaje según el modelo de entornos convergentes y se desarrollan los ejes de acción. Se socializan las intenciones educativas, se suscribe un contrato didáctico y se determina la forma de evaluación.

La tercera fase hace alusión al tipo de acompañamiento y a los procesos de seguimiento a las actividades planteadas, registrando las principales dificultades para llevar a cabo las tareas, y se establecen formas de superarlas.
Una cuarta fase evalúa el proceso de formación frente a los contextos mencionados en la primera fase.

El examen de los procesos adelantados en las diferentes fases se realizó teniendo en cuenta las siguientes categorías, bajo un análisis del contenido.

Tabla 1. Categorías de análisis

\begin{tabular}{|c|l|}
\hline \multicolumn{1}{|c|}{ CATEGORÍA } & \multicolumn{1}{c|}{ DESCRIPTORES } \\
\hline MatemaTIC en los diferentes contextos de formación & $\begin{array}{l}\text { Identifica los principales ejes problémicos que obstaculizan el desarrollo de las } \\
\text { competencias en matemáticas en relación con otros contextos. } \\
\text { Relaciona los ejes problémicos de la profesión para el diseño de las SDD. }\end{array}$ \\
\hline MatemaTIC dentro de la estructura didáctica & $\begin{array}{l}\text { Ubica la estrategia dentro del modelo de entornos convergentes. } \\
\text { Establece rasgos distintivos para delimitar su accionar. } \\
\text { Evalúa su progreso según las condiciones didácticas. }\end{array}$ \\
\hline
\end{tabular}

Fuente. Elaboración propia.

\section{Resultados}

A continuación, se presentan de forma condensada algunos resultados con relación a las fases metodológicas desarrolladas.

De la primera fase se puede exaltar que, durante el periodo analizado, MatemaTIC se ofertó a estudiantes que se encontraban en diversas regiones del país, según la distribución presentada en la figura 1. De esta población, y después de aplicar una prueba estandarizada, se pudo determinar que el número de respuestas acertadas a problemas relacionados con tres de los pensamientos matemáticos valorados — numérico, variacional y aleatorio- fue bajo, como se muestra en la tabla 2. 


\begin{tabular}{|c|c|}
\hline Pensamiento & Porcentaje de aciertos \\
\hline Numérico & $20 \%$ \\
\hline Variacional & $14 \%$ \\
\hline Aleatorio & $17 \%$ \\
\hline
\end{tabular}

Fuente. Elaboración propia.

Con base en esto se establece que cualquiera de los tres pensamientos puede ser abordado durante el diseño de las unidades didácticas, pero se determina que bajo criterios disciplinares y didácticos el pensamiento numérico debe primar. Es imperativo mostrar que aun cuando los pensamientos evaluados no constituyen el total de los requerimientos teóricos especificados, sí muestran las rutas de implementación y de acción a desarrollar.

Una vez determinado el pensamiento matemático por intervenir, se establecen los ejes problémicos de la profesión en los cuales se puede tener incidencia; entre ellos se destacan problemas de tipo financiero -créditos, inversiones, tasas de interés, etc.-, económico — tasas, devaluación, divisas, IPC, etc. - y de mercadeo — ventas, registros, inventarios, etc.- - Los problemas seleccionados se relacionaron con las ventas y el índice de precio al consumidor (IPC); para ello se utilizan fuentes teóricas institucionales nacionales, como el Departamento Administrativo Nacional de Estadística (DANE), el Banco de la Republica y los ministerios.

En las siguientes fases metodológicas se incorpora el problema a los ejes de acción, de acuerdo con la disponibilidad de los entornos de aprendizaje, y se establece el tipo de seguimiento a realizar, como se aprecia en la tabla 3. 
Tabla 3. Desarrollo metodológico relacionado con las fases 2, 3 y 4

\begin{tabular}{|c|c|c|c|}
\hline EJE DE ACCIÓN & ACTIVIDAD PRINCIPAL DE APRENDIZAJE & ENTORNO PREDOMINANTE & ACTIVIDAD DE SEGUIMIENTO \\
\hline Indagación & $\begin{array}{l}\text { Lectura o video referente al tema de origen institucional } \\
\text { nacional y participación en foro de discusión bajo unas } \\
\text { preguntas orientadoras }\end{array}$ & Aula virtual & Respuesta a foro y discusión \\
\hline Problematización & $\begin{array}{l}\text { Presentación de la situación problémica relacionada } \\
\text { con la profesión en donde intervienen diferentes clases } \\
\text { de números, representaciones y registros. Así mismo, } \\
\text { se formulan preguntas }\end{array}$ & Teleclase & $\begin{array}{c}\text { Control de asistencia y de } \\
\text { participación }\end{array}$ \\
\hline Tematización & $\begin{array}{l}\text { Desarrollo de los contenidos propios referentes a } \\
\text { números, en especial a racionales, operaciones, } \\
\text { cambio de registro y representación - porcentajes y } \\
\text { gráficas- }\end{array}$ & Módulo digital y pregrabados & Tutorías académicas \\
\hline Aplicación & $\begin{array}{l}\text { Comparación de las diferentes formas de solucionar } \\
\text { la situación problémica original en relación con el } \\
\text { referente conceptual y procedimental anterior }\end{array}$ & $\begin{array}{c}\text { Aula virtual, compartir material en } \\
\text { línea - videos, presentaciones o } \\
\text { trabajos escritos- }\end{array}$ & Valoraciones de procesos \\
\hline Finalización & $\begin{array}{l}\text { Validación de respuestas y procedimientos dados en } \\
\text { la aplicación }\end{array}$ & Teleclase & Valoración de procesos \\
\hline
\end{tabular}

Fuente. Elaboración propia.

Después de llevar a cabo el proceso descrito y de aplicar una prueba de control final, se pudo establecer una mejoría en los resultados a una prueba final que superó el $79 \%$ de aprobación. De igual forma, es prudente reconocer que el $90 \%$ de los estudiantes finalizaron MatemaTIC e independiente del resultado final, consideraron que MatemaTIC aportó a su desarrollo profesional y de manejo de plataforma.

\section{Conclusiones}

MatemaTIC nació como una estrategia exclusiva para fomentar el desarrollo y la consolidación de competencias en matemáticas; no obstante, dados los escenarios cambiantes y la movilidad de los estudiantes, ha presentado modificaciones relevantes, entre las cuales destacan las que señalamos a continuación.
En primer lugar, MatemaTIC se acoge a la familia de problemas de la profesión, en este sentido, las situaciones utilizadas en las secuencias didácticas digitales están en sintonía con los problemas profesionales o académicos de su área. Esta relación hace que los estudiantes, por un lado, establezcan nexos funcionales entre el campo teórico de las 
matemáticas y los campos de aplicación —Administración de Empresas-, y, por el otro, que durante los diseños de las unidades didácticas contextuales el profesor deba actualizar de forma permanente sus conocimientos más allá de lo disciplinar.

Es preciso mencionar que durante la aplicación de MatemaTIC en el periodo 2018-1 se experimentó sobre problemas referentes a las ventas y el IPC, sus indicadores y los índices que estos aportaban para la toma de decisiones, los cuales se relacionaron con cambios representacionales en matemáticas, transformaciones funcionales y modelamiento de problemas sobre una base numérica, aleatoria y variacional.

De igual forma, la estrategia se materializó de acuerdo con las disposiciones institucionales y el modelo de entornos convergentes del Departamento de Estudios a Distancia, lo que ha permitido una construcción sistemática de unidades didácticas contextuales con diferentes grados de complejidad y de diseño neutral. Para convertirse, así, en un elemento de inclusión académica dada la diversidad cultural y sociodemográfica de los estudiantes, y que estos amplíen competencias tanto matemáticas como de su futura profesión. Es de anotar que el momento de indagación es fundamental para evaluar el avance en la competencia matemática, puesto que, al elaborar un diagnóstico real, MatemaTIC puede hacer una intervención inmediata sobre los aspectos que podrían dificultar el progreso en su plan de estudios.
Se debe advertir que MatemaTIC presenta mejores resultados en los periodos académicos iniciales de los programas de estudio, ya que su andamiaje didáctico, además de contribuir a la consolidación de competencias, incorpora gradualmente las Tecnologías de la Información y la Comunicación en el trabajo académico individual y grupal. De igual forma, cuando MatemaTIC hace seguimiento académico, lo realiza sobre la base del mejoramiento individual, que contribuye a institucionalizar los procesos de regulación metacognitiva en los estudiantes, lo que posibilita la competencia de aprender a aprender.

Por último, es importante aclarar que MatemaTIC actúa según las disposiciones institucionales, las necesidades de la población estudiantil y el grado de complejidad cambia de acuerdo con las dinámicas grupales. 


\section{Referencias}

Ágamez, J., Daza, C., Rincón, A. (2018). Co-creación de unidades didácticas contextualizadas y secuencias didácticas digitales en el modelo de entornos convergentes MEC-Complexus. En M. Coronado-Gallardo; R. Morales-Gamboa; A.Y. Ávila Moreno (Coord.) Escenarios creativos para la educación. (Tomo I, 65-88). Guadalajara: Universidad de Guadalajara. Recuperado de https://bit.ly/3gnMoeZ

Ágamez, J., Rincón, A., Silva, L. (2017). Secuencias didácticas digitales en el modelo de entornos convergentes MECComplexus. Recuperado de https://bit.ly/3mKcr2f

Coll, C. S. (1988) Significado y sentido del aprendizaje escolar. Reflexiones en torno al concepto de aprendizaje significativo. Infancia y Aprendizaje, 11(41), 131-142. DOI: https://doi.org/ 10.1080/02103702.1988.10822196

Dávila, M.; Borrachero, A.; Cañada, F.; Sánchez, J. (2018). Factores afectivos y cognitivos en el aprendizaje de los cambios físicos y químicos de la materia en alumnos de Educación Secundaria. Tecné, Episteme y Didaxis, (44), 91-110.

Llinares, S. (2008). Construir el conocimiento necesario para enseñar matemática. Evaluación e Investigación, (1), 9-30.

Melgar, M. y Elisondo, R. (2017). Metacognición y buenas prácticas en la universidad. ¿Qué aspectos valoran los estudiantes? Innovación educativa, 17(74),17-38.

Ministerio de Educación Nacional - MEN. (2006). Estándares básicos de competencias en matemáticas Ministerio de Educación Nacional. Recuperado de https://bit.ly/2lgFewk
Ministerio de Educación Nacional - MEN. (Septiembre de 2010). Lineamientos para la articulación de la educación media. Recuperado de https://bit.ly/37ypMo0

Sarrionandia, E. y Duk, C. (2008). Inclusión educativa. REICE. Revista Iberoamericana sobre Calidad, Eficacia y Cambio en Educación, 6(2),1-8.

Segura, M. (2011). Plataformas educativas y redes docentes. En R. Carneiro; J. C. Toscano; T. Díaz (Coord.) Los desafíos de las TIC para el cambio educativo. (94-110). Madrid: Organización de Estados Iberoamericanos para la Educación, la Ciencia y la Cultura (OEI).

Yanes, J. (2014). Las TIC y la crisis de la educación. Algunas claves para su comprensión. Chile: Biblioteca Digital Educa. Recuperado de https://bit.ly/3qC9gMv 